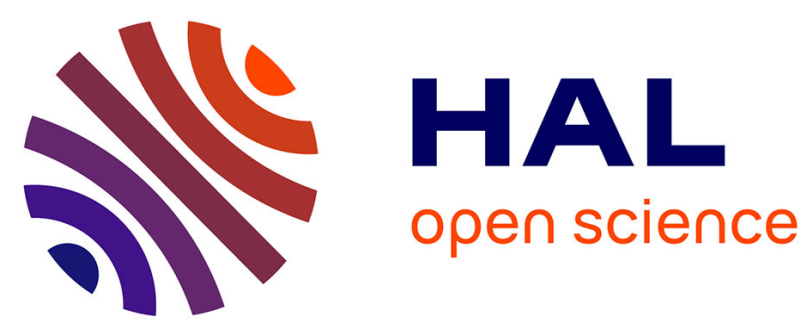

\title{
Apport du Multimorph à l'étude des processus de Reconnaissance Émotionnelle Faciale (REF). Exemple de la personnalité borderline à l'adolescence
}

M. Robin, S. Berthoz, G. Kedia, C. Dugre-Le Bigre, F. Curt, M. Speranza, D. Sapinho, A. Pham-Scottez, M. Corcos

\section{To cite this version:}

M. Robin, S. Berthoz, G. Kedia, C. Dugre-Le Bigre, F. Curt, et al.. Apport du Multimorph à l'étude des processus de Reconnaissance Émotionnelle Faciale (REF). Exemple de la personnalité borderline à l'adolescence. Annales Médico-Psychologiques, Revue Psychiatrique, 2011, 169 (2), pp.120. 10.1016/j.amp.2010.12.002 . hal-00727592

\section{HAL Id: hal-00727592 \\ https://hal.science/hal-00727592}

Submitted on 4 Sep 2012

HAL is a multi-disciplinary open access archive for the deposit and dissemination of scientific research documents, whether they are published or not. The documents may come from teaching and research institutions in France or abroad, or from public or private research centers.
L'archive ouverte pluridisciplinaire HAL, est destinée au dépôt et à la diffusion de documents scientifiques de niveau recherche, publiés ou non, émanant des établissements d'enseignement et de recherche français ou étrangers, des laboratoires publics ou privés. 


\section{Accepted Manuscript}

Title: Apport du Multimorph à l'étude des processus de Reconnaissance Émotionnelle Faciale (REF). Exemple de la personnalité borderline à l'adolescence

Authors: M. Robin, S. Berthoz, G. Kedia, C. Dugre-Le Bigre, F. Curt, M. Speranza, D. Sapinho, A. Pham-Scottez, M. Corcos

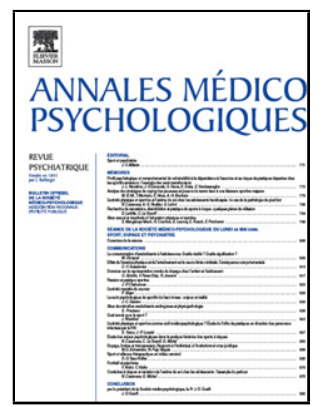

PII: S0003-4487(10)00408-7

DOI: $\quad$ doi:10.1016/j.amp.2010.12.002

Reference: $\quad$ AMEPSY 1280

To appear in: Annales Médico-Psychologiques

Please cite this article as: Robin M, Berthoz S, Kedia G, Bigre CD-L, Curt F, Speranza M, Sapinho D, Pham-Scottez A, Corcos M, Apport du Multimorph à l'étude des processus de Reconnaissance Émotionnelle Faciale (REF). Exemple de la personnalité borderline à l'adolescence, Annales medio-psychologiques (2010), doi:10.1016/j.amp.2010.12.002

This is a PDF file of an unedited manuscript that has been accepted for publication. As a service to our customers we are providing this early version of the manuscript. The manuscript will undergo copyediting, typesetting, and review of the resulting proof before it is published in its final form. Please note that during the production process errors may be discovered which could affect the content, and all legal disclaimers that apply to the journal pertain. 
Communication

Apport du Multimorph à l'étude des processus de Reconnaissance Émotionnelle Faciale (REF). Exemple de la personnalité borderline à l'adolescence Use of the Multimorph in Facial Emotion Recognition.

Example of Borderline Personality in Adolescence

M. Robin ${ }^{\text {a,b,c,* }}$, S. Berthoz ${ }^{\text {a,b,c }}$, G. Kedia ${ }^{\text {d }}$, C. Dugre-Le Bigre ${ }^{\text {a,b,c }}$, F. Curt ${ }^{\text {a,b,c }}$, M. Speranza ${ }^{\text {e }}$, D. Sapinho ${ }^{\text {, }}$, A. Pham-Scottez ${ }^{\text {b,f }}$, M. Corcos ${ }^{\text {a,b,c }}$

${ }^{a}$ Institut Mutualiste Montsouris, Paris, France

${ }^{b}$ INSERM U 669, France

${ }^{c}$ Université Paris Descartes Paris V et Paris XI, France

${ }^{d}$ Université de Cologne, Allemagne

${ }^{e}$ Hôpital Mignot, Versailles, France

${ }^{f}$ Hôpital Sainte-Anne, Paris, France

Auteur correspondant : M. Robin, IMM, 42, boulevard Jourdan, 75014 Paris, France Tél. : 0156616919

Adresse email : marion.robin@imm.fr

\section{Résumé}

Cet article expose l'intérêt du Multimorph, outil expérimental évaluant les performances de traitement dynamique de visages émotionnels par le morphing. La version présentée ici permet de voir évoluer lentement et continuellement un visage de la neutralité à l'une des six émotions de base pleinement exprimée (colère, dégoût, joie, peur, surprise et tristesse). L’intérêt du Multimorph est analysé à partir d'une revue des études utilisant ce paradigme dans la mesure des capacités de reconnaissance émotionnelle faciale. L'exemple de la personnalité borderline à l'adolescence est développé, afin d'en analyser les corrélats clinico-expérimentaux. Les limites et les perspectives du Multimorph sont ensuite discutées.

Mots clés : Adolescence ; Multimorph ; Personnalité Borderline ; Reconnaissance Émotionnelle Faciale

\section{Abstract}

An increasing interest in the study of facial emotion recognition in many psychiatric disorders has emerged over the past few years. The morphing technique allowed opening new 
perspectives in experimentation, by using dynamic expressions of emotions, such as in the Multimorph task. Based on a literature review, we present here the interest of the Multimorph task. It includes 36 trials where a neutral (0 \%) face expresses increasing degrees of emotional intensity, slowly changing to a full-blend (100\%) emotion. Then, the example of borderline personality disorder in adolescence is developed, to examine how the observed pattern of results could account for the reported clinical characteristics. Limitations and prospects of the Multimorph are further discussed.

Keywords: Adolescence; Borderline Personality Disorder; Facial Emotion Recognition; Multimorph

\section{Introduction}

L’expression émotionnelle faciale témoigne de l'état affectif du sujet mais aussi de ses intentions et tendances à l'action [9,12]. Confronté à ces expressions, l'interlocuteur qui reçoit l'information régule ses propres états affectifs et adapte son comportement. Il s’agit donc de signaux majeurs de l'interaction sociale. D’un point de vue expérimental, l'analyse des processus cognitifs et affectifs impliqués dans les interactions sociales montre un nombre croissant de données en faveur d'anomalies de la Reconnaissance Émotionnelle Faciale (REF) dans les troubles psychiatriques [2,13,14]. Initialement évaluée par des stimuli photographiques (évaluation qualitative), la REF peut aujourd’hui être analysée de façon dynamique et fine (quantitative) [5]. Ainsi, le Multimorph est une tâche de REF comportant une série de visages évoluant lentement mais continuellement, de la neutralité vers l'une des six émotions de base pleinement exprimée. Ce paradigme permet d'estimer les capacités de REF à des niveaux inférieurs d'intensité du stimulus, et d'accéder ainsi à des déficits plus subtils. Nous présentons ici une version adaptée du paradigme développé par l'équipe de James Blair [4], complété par une tâche contrôle (MultiForm).

\section{Instrument}

\subsection{Multimorph}


Nous avons adapté une version du Multimorph comprenant 36 essais randomisés (six visages : trois hommes et trois femmes, six émotions par visage), à partir des photographies validées d'Eckman et Friesen (1976). Quarante images sont présentées de façon continue sur 20 secondes. Le participant a pour consigne d'identifier l'émotion présentée par le visage dès qu'il la reconnaît. Il peut changer d'avis autant de fois qu'il le souhaite avant la fin de l'essai, et il lui est demandé de donner son avis définitif à la fin. Une épreuve d'entraînement a été réalisée selon un protocole comparable.

Figure 1 : Multimorph. Exemple du stimulus « peur » exprimé par un visage d’homme.

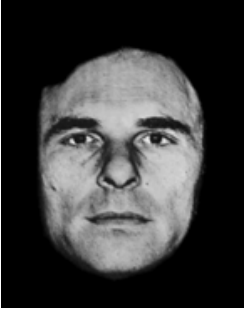

Neutralité
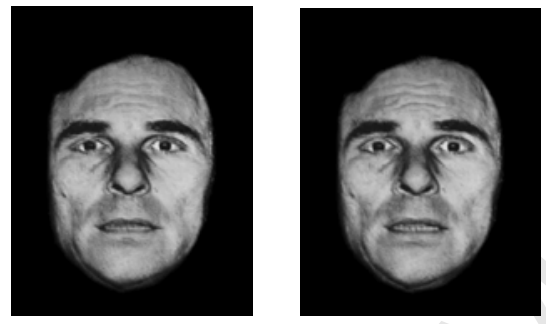
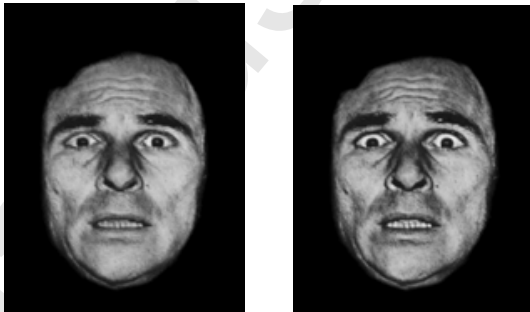

$100 \%$

\subsection{Scores au Multimorph}

La mesure de la performance de reconnaissance émotionnelle finale est d'abord déterminée par le pourcentage de réponses correctes à la fin de l'essai, i.e., le « taux de succès » lorsque 100 \% de l'émotion est exprimée.

La mesure de la sensibilité est déterminée par le délai (nombre d’images) de la première identification correcte pour chaque émotion, i.e., le « délai du succès ».

De plus, différentes variables peuvent être étudiées à partir des erreurs (taux d'échecs précoces, délai de premières réponses fausses) et des matrices de confusion (biais de reconnaissance en faveur de certaines émotions).

\subsection{MultiForm}

Le MultiForm a été conçu comme épreuve motrice et attentionnelle contrôle, selon un protocole identique à partir de formes géométriques (six formes, 36 essais).

\section{Résultats des principales études}


Jusqu’à présent, le Multimorph a été essentiellement utilisé dans le cadre des troubles de la personnalité où il trouve effectivement un grand intérêt, étant donné le caractère central du dysfonctionnement relationnel dans ces cas.

À l’origine utilisé dans une population d'enfants présentant des traits psychopathiques, le Multimorph a permis de mettre en évidence un déficit de reconnaissance de la peur ainsi qu'une diminution de la sensibilité à la tristesse en comparaison à des sujets témoins. Plus récemment, dans une population d'adultes borderline, le Multimorph a permis de montrer que les patients borderline montraient une sensibilité supérieure aux sujets témoins à la joie et à la colère [17]. La réalisation d'un paradigme comparable, mais avec une moindre sensibilité et dans une population plus comorbide (notamment avec l'état de stress post-traumatique) a montré que les adultes borderline présentaient des seuils de sensibilité comparables aux témoins [7]. Ainsi, la sensibilité de l'outil paraît majeure, et la sévérité clinique de l'échantillon représenterait bien un facteur d'influence de la REF.

L’étude que nous avons réalisée dans la lignée de ces résultats portait sur les capacités de REF d'adolescents borderline [19-22]. L'adjonction d'une tâche motrice et attentionnelle représente un apport supplémentaire vis-à-vis des études précédentes.

\section{L’exemple de la personnalité borderline adolescente}

L’impulsivité, la labilité du fonctionnement émotionnel et la survenue d’accès de colère chez les patients borderline sont à l'origine d'une gêne notable dans leurs interactions sociales. Linehan avance l'idée que ces difficultés affectives correspondent au noyau dysfonctionnel à l'origine du trouble, et que l’hyperréactivité émotionnelle borderline est liée chez ces patients à une difficulté à identifier les stimuli émotionnels de l'environnement $[17,23]$.

Certaines études utilisant des images statiques réalisées chez l'adulte ont effectivement montré un déficit de reconnaissance de la colère, de la peur et du dégoût, ou bien de la colère, de la tristesse et de la peur, en comparaison à des sujets témoins $[3,8,15]$. Wagner rapporte que les femmes borderline ont un déficit de reconnaissance de la neutralité émotionnelle, qu'elles ont tendance à «sur-identifier » comme étant de la peur par rapport aux témoins [23]. Très peu d’études avaient été réalisées chez l’adolescent, et toujours avec des stimuli statiques, montrant que les adolescents borderline avaient plus de difficultés à identifier l’intensité émotionnelle que la qualité propre de l’émotion [6]. 
L’étude cas-témoins réalisée par notre équipe portait sur une population de 22 adolescents présentant une personnalité borderline selon les critères du DSM-IV-TR, majoritairement hospitalisés [1,22]. Nous avons observé que les sujets borderline obtenaient des taux de succès comparables à ceux des témoins. En revanche, l'analyse des délais de succès a montré que les adolescentes borderline mettaient plus de temps à reconnaître la colère et la joie que les témoins. La comparaison des erreurs aux temps précoces (taux et délais) n’a pas montré de différences intergroupes. Les résultats montrent donc que les adolescentes borderline présentent, comparativement aux témoins, une plus faible capacité globale de REF. Point important : celle-ci est due à une plus grande difficulté à identifier une émotion lorsqu'elle est exprimée à de faibles degrés d'expressivité, mais il n’y a pas de déficit pour reconnaître l'émotion pleinement exprimée. En revanche, les sujets borderline n’ont pas plus d'erreurs précoces, c'est-à-dire que leur déficit ne peut s'expliquer par une plus grande impulsivité expérimentale [19-22].

Nos résultats sont exploratoires dans le domaine de l'adolescence. La nature des résultats souligne l'importance d’un paradigme dynamique pour mettre en évidence de subtils déficits, y compris dans des échantillons ayant, comme dans notre étude, un certain niveau de sévérité clinique.

\section{Discussion - Intérêt de l’outil}

L’intérêt principal du Multimorph est d’être dynamique, donc plus écologique. Bien que l'expression émotionnelle soit plus lente que dans la situation naturelle entre deux interlocuteurs humains, il représente un compromis extrêmement utile, dans la mesure où il permet d'échapper à une évaluation purement statique et qualitative ; en même temps, le participant a la possibilité d'exprimer ses hésitations et doutes au fur et à mesure que l'émotion devient de plus en plus intense et évidente.

Les résultats obtenus dans les échantillons étudiés, principalement les troubles de personnalité, montrent qu'il existe des corrélats clinico-expérimentaux prometteurs. Nos résultats chez l'adolescent vont dans le sens de l'hypothèse de Linehan, en décrivant des difficultés de REF, qui pourraient effectivement être liées à l’hyperréactivité émotionnelle borderline, notamment autour de la colère et de la joie. Cependant, l'utilisation du paradigme dans d'autres catégories nosographiques pourrait également apporter des données psychopathologiques importantes et permettre de confirmer l'aspect de plus en plus transnosographique de ce type de résultats en psychopathologie psychiatrique. 
L'utilisation de cet instrument sur des populations cliniques pose la question de l'interaction avec les traitements psychotropes. La recherche en sciences cognitives en population clinique rencontre, avec la fréquente et nécessaire prescription de psychotropes, l'une de ses principales difficultés. Cependant, la solution que trouveraient ces recherches dans la sélection de patients non ou moins traités représenterait une perte de validité clinique évidente. Ainsi, le développement de recherches dites «naturalistes » vise à rester au plus près de la clinique quotidienne. La pathologie borderline est, à ce titre, un exemple de la stérilité d’une démarche de sélection d’une forme "pure » (homogénéité des cas, absence de comorbidité, de traitement) de la pathologie. En revanche, l'établissement de tâches contrôles, l'analyse des résultats en sous-groupes, l'analyse des facteurs de confusion éventuels, permettent d'affiner les résultats, de poser des hypothèses pertinentes pour les études suivantes. Dans le cas des adolescents borderline, l'étude des délais de réponse à la tâche contrôle a permis de montrer que l'effet des traitements psychotropes ne pouvait pas expliquer la différence de REF intergroupe.

La tâche que représente le Multimorph est facile à comprendre et ludique pour le participant. Elle nécessite un matériel informatique relativement simple. Le temps de passation est court, ce qui augmente l'attractivité et donc la faisabilité de l'étude.

Enfin, un des intérêts principaux des résultats des études portant sur la REF est à rechercher du côté de la thérapeutique. La compréhension neuroscientifique des caractéristiques cliniques de la personnalité borderline, à savoir la difficulté à traiter les stimuli sociaux, est d'un intérêt particulier dans le domaine des psychothérapies. La difficulté des sujets borderline à interpréter les stimuli faciaux ambigus et la tendance à les interpréter du côté de la menace apportent à la psychanalyse des éléments pour penser la dynamique de transfert avec un nouvel éclairage. Le processus d’identification projective pourrait être ici compris comme un processus adaptatif où le sujet attribue à autrui ses états affectifs internes, en réponse à une difficulté à se représenter exactement les états émotionnels d'autrui. Les difficultés perceptuelles des patients borderline font l'objet d'un développement actuel de certaines formes de psychothérapies, dans lesquelles le travail porte, entre autres, sur la capacité à différencier un signal social ambigu ou subtil [11,16].

\section{Conclusion et perspectives}

À l'heure où la recherche en psychologie sociale se centre très précisément sur l'émotion telle qu'elle est identifiée dans le cadre de la relation interindividuelle, le paradigme 
du Multimorph montre tout son intérêt. Les résultats obtenus dans différentes populations cliniques, et notamment chez les adolescents borderline, montrent que le Multimorph est suffisamment sensible pour discriminer les processus de reconnaissance de l'émotion chez les patients et les sujets sains. Il ouvre également la voie à une exploration transnosographique, à la mise en évidence de traits infracliniques, permettant ainsi de poser de nouvelles perspectives théoriques et cliniques, voire thérapeutiques. Enfin, le développement de cet outil en version française permettra, nous l'espérons, d'étendre son utilisation à la communauté francophone, et de poursuivre les travaux déjà réalisés par les équipes anglosaxonnes.

\section{Conflit d'intérêt : aucun.}

\section{Références}

[1] American Psychiatric Association. Diagnostic and Statistical Manual of Mental Disorders, Fourth Edition, Text Revision (DSM-IV-TR). Washington DC: APPI; 2000.

[2] Bolte S, Poustka F. The recognition of facial affect in autistic and schizophrenic subjects and their first degree relatives. Psychol Med 2003;33:907-15.

[3] Bland AR, Williams CA, Scharer K, Manning S. Emotion processing in borderline personality disorders. Issues Ment Health Nurs 2004;25:655-72.

[4] Blair RJ, Colledge E, Murray L, Mitchell DG. A selective impairment in the processing of sad and fearful expressions in children with psychopathic tendencies. J Abnorm Child Psychol 2001;29:491-8.

[5] Calder AJ, Young AW, Perrett DI, Etcoff NL, Rowland D. Categorical perception of morphed facial expressions. Visual Cognition 1996;3:81-117.

[6] Ceumern-Lindenstjerna IA, Brunner R, Parzer P, Frey M, Fiedler P, Resch F. Perception of emotional facial expressions in female adolescents with borderline personality disorder. Z Kinder Jugendpsychiatr Psychother 2007;35:333-40.

[7] Domes G, Czieschnek D, Weidler F, Berger C, Fast K, Herpertz SC. Recognition of facial affect in Borderline Personality Disorder. J Person Disord 2008;22:135-47.

[8] Domes G, Schulze L, Herpertz SC. Emotion recognition in borderline personality disorder-a review of the literature. J Person Disord 2009;23:6-19.

[9] Ekman P. Emotion in the human face (2nd ed.). Cambridge: Cambridge University Press; 1982. 
[10] Ekman P, Friesen, WV. Pictures of facial affect 1976. Available from http://www.pauleckman.com/research_cds.php

[11] Fonagy P, Bateman AW. Mechanisms of change in mentalization-based treatment of BPD. J Clin Psychol 2006;62:411-30.

[12] Frijda NH. The emotions. Cambridge: Cambridge University Press; 1986.

[13] Kohler CG, Turner TH, Bilker WB, Brensinger CM, Siegel SJ, Kanes SJ, et al. Facial emotion recognition in schizophrenia: intensity effects and error pattern. Am J Psy 2003;160:1768-74.

[14] Leppanen JM. Emotional information processing in mood disorders: A review of behavioral and neuroimaging findings. Curr Op Psy 2006;19:34-9.

[15] Levine D, Marziali E, Hood J. Emotion processing in borderline personality disorders. J Nerv Ment Dis 1997;185:240-6.

[16] Linehan MM. Cognitive-behavioral treatment of borderline personality disorder. New York: Guilford Press; 1993.

[17] Linehan MM. Understanding borderline personality disorder. New York: Guilford Press; 1995.

[18] Lynch TR, Rosenthal MZ, Kosson DS, Cheavens JS, Lejuez CW, Blair RJ. Heightened sensitivity to facial expressions of emotion in borderline personality disorder. Emotion 2006;6:647-55.

[19] Robin M. Capacités de reconnaissance émotionnelle faciale des adolescents borderline : étude comparative. Master Recherche Neurosciences. Université Paris VI. Sous la direction du Pr Corcos et du Dr Pham-Scottez, 2007, 21p.

[20] Robin M, Berthoz S, Pham-Scottez A, Kedia G, Curt F, Dugre Le Bigre C, Corcos M. Lower sensitivity to facial expressions of anger and happiness in adolescents with borderline personality disorder. Berlin: Communication orale présentée au $1^{\mathrm{er}}$ congrès sur la Personnalité Borderline; juillet 2010.

[21] Robin M, Pham-Scottez A, Corcos M. Emotional dysregulation in adolescents with BPD-use of the multimorph facial affect recognition task. San Diego: Communication présentée au 160 è congrès de l'American Psychiatric Association; mai 2007.

[22] Robin M, Pham-Scottez A, Curt F, Dugre-Le Bigre C, Sapinho D, Corcos M, et al. Lower Sensitivity to Facial Expressions in Adolescents with Borderline Personality Disorder (soumis). 
[23] Wagner AW, Linehan MM. Facial expression recognition ability among women with borderline personality disorder: implications for emotion regulation? J Pers Disord 1999;13:329-44.

\section{Discussion}

Pr J.-M. Vanelle - Une remarque et deux questions: Akiskal a pu différencier un groupe de bipolaires dans une population de malades borderline. Il semblerait que la gestion émotionnelle ne permette pas aujourd’hui de les différencier.

Les critères des personnalités borderline que vous utilisez sont ceux du DSM-IV-TR. Leur caractère grossier, basé sur des comportements plus que sur la psychopathologie avec les notions d'anaclitisme ou de tendance interprétative ne constitue-t-il pas une importante limite?

Dans votre exploration de l'humeur, prenez-vous en compte la simple humeur dépressive ou recherchez-vous un état dépressif caractérisé?

Réponse du Rapporteur - Les études expérimentales portant sur la psychopathologie psychiatrique mettent, dans la grande majorité des cas, en évidence des traits, des phénomènes infracliniques qui sont transnosographiques, réinterrogeant ainsi la validité de nos catégories diagnostiques. Effectivement, les études portant sur la reconnaissance émotionnelle faciale décrivent des difficultés de reconnaissance sur plusieurs catégories diagnostiques. Ainsi, l'intérêt est d'explorer plus avant les particularités de chaque déficit (type d'émotions, déficit ou hypersensibilité), mais aussi d’établir des corrélations avec des traits psychopathologiques fins, comme la dimension d'anaclitisme, le sentiment d'intrusion ou l'intensité du vécu émotionnel (d’une façon générale ou au moment de l'expérimentation). Dans notre étude chez les adolescents, la prise en compte d'une comorbidité dépressive (mesurée par les critères DSM-IV-TR de l'état dépressif majeur) a permis d'affiner les résultats dans le sens de difficultés plus grandes de reconnaissance émotionnelle faciale chez les adolescents borderline déprimés versus les adolescents borderline non déprimés. Mais d’autres études ont analysé la dépressivité sur un mode dimensionnel ou la tristesse au moment de l’expérimentation.

$\operatorname{Pr}$ B. Lafont - Est-il possible de préciser quelles conséquences pratiques dans la prise en charge de ces patients on peut déjà entrevoir à partir de ces données ? 
Réponse du Rapporteur - Dans le cadre d'un bilan clinique, nous pouvons penser que l'analyse pour un patient donné, de ses capacités de reconnaissance émotionnelle, pourrait permettre de préciser ce qui entrave le comportement social du patient, et avec quelle intensité il est entravé à ce niveau.

De plus, les apports expérimentaux récents sur la reconnaissance émotionnelle faciale ont déjà permis l'adaptation de certaines psychothérapies centrées sur l’identification des signaux sociaux, chez l'adulte ayant une personnalité borderline notamment. La technique psychanalytique trouve probablement ici des éléments supplémentaires pour comprendre le phénomène psychothérapique qu'est la psychose de transfert, découverte justement à l'occasion de traitements de patients états limites, mis en position allongée dans le cadre de la cure classique (donc en situation d'ambiguïté perceptive).

Nos résultats chez l'adolescent borderline confirment probablement l'intérêt que les psychothérapies peuvent avoir dans cette population clinique, et notamment autour de l'identification de la colère d'autrui, du vécu de sa colère propre, et de la confusion entre ces deux aspects, que ce soit par la mentalisation, la dialectique, ou par le jeu du psychodrame. 